\title{
Statistical Conformation of Human Plasma Fibronectin
}

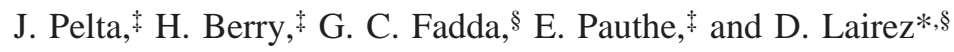 \\ ERRMECE, Université de Cergy-Pontoise, 95302 Cergy-Pontoise Cedex, France, and Laboratoire Léon Brillouin, \\ CEA-CNRS, CEA/Saclay, 91191 Gif-sur-Yvette Cedex, France
}

Received December 3, 1999

\begin{abstract}
Fibronectin is a multifunctional glycoprotein (molecular mass, $M=530 \mathrm{~kg} / \mathrm{mol}$ ) of the extra cellular matrix (ECM) having a major role in cell adhesion. In physiological conditions, the conformation of this protein still remains debated and controversial. Here, we present a set of results obtained by scattering experiments. In "native" conditions, the radius of gyration $\left(R_{\mathrm{g}}=15.3 \pm 0.3 \mathrm{~nm}\right)$ was determined by static light scattering as well as small-angle neutron scattering. The hydrodynamic radius $\left(R_{\mathrm{H}}=11.5 \pm\right.$ $0.1 \mathrm{~nm}$ ) was deduced from quasi-elastic light scattering measurements. These results imply a low internal concentration $\left(M / R_{\mathrm{g} / \mathrm{H}}^{3}\right)$ compared to that of usual globular proteins. This is also confirmed by the ratio $R_{\mathrm{H}} / R_{\mathrm{g}}=0.75 \pm 0.02$ consistent with a Gaussian chain, whereas $R_{\mathrm{H}} / R_{\mathrm{g}}=1.3$ for spherical shaped molecules. However, adding a denaturing agent (urea $8 \mathrm{M}$ ) increases $R_{\mathrm{g}}$ by a factor 2 . This means that fibronectin "native" chain is not either completely unfolded. The average shape of fibronectin conformation was also probed by small-angle neutron scattering performed for reverse scattering vector $q^{-1}$ smaller than $R_{\mathrm{g}}(0.2$ $<q^{-1}<15 \mathrm{~nm}$ ). The measured form factor is in complete agreement with the form factor of a random string of 56 beads of $5 \mathrm{~nm}$ diameter. It rules out the possibility of unfolded chain as well as globular structures. These results have structural and biological implications as far as ECM organization is concerned.
\end{abstract}

Fibronectin is a multifunctional glycoprotein expressed by many different cell types (hepatocytes, fibroblasts, macrophages, and leukocytes among others). It is found in plasma and extracellular matrix (ECM). ${ }^{1}$ Its main biological functions are based on its affinity for different biological compounds such as cell surface receptors, ECM proteins (collagens, heparin, etc), DNA, gangliosides, and immunoglobulins. In vivo, fibronectin mediates cell adhesion to the ECM, and contributes to phagocytosis regulation, wound healing, cell proliferation, differentiation, and locomotion (1).

Fibronectin is composed of two identical polypeptide subunits covalently linked via two disulfide bonds near their carboxy termini. Each subunit is made of nearly 2500 amino acids (molecular weight about 250000 ). It consists of the repetition of 56 modules (1) of three types having a welldefined structure and a high degree of internal homology. Modules of type I, II, and III consist of about 45, 60, and 90 amino acids, respectively. The type I modules make up the $\mathrm{NH}_{2}$-terminal and $\mathrm{COOH}$-terminal ends of the polypeptide. Two type-II modules interrupt a row of nine type-I modules at the $\mathrm{NH}_{2}$-terminus. Fifteen type-III modules, make up the middle of the polypeptide $(1,2)$. Type-I, II, and III modules are also found in many other proteins, type III even being ubiquitous in animal proteins (3). These modules are connected together by short polypeptide segments sensitive to proteinases and are grouped in functional domains that

* To whom correspondence should be addressed: Phone: (33) 1 690872 31. Fax: (33) 1690882 61. E-mail: lairez@cea.fr.

$\doteqdot$ Université de Cergy-Pontoise.

§ Laboratoire Léon Brillouin.

${ }^{1}$ Abbreviations: ECM, extra cellular matrix; NMR, nuclear magnetic resonance; DCl, deuterium chloride; EDTA, ethylenediaminetetraacetic acid. express specific binding activities (4). The above structure of fibronectin varies somewhat depending on the cellular source of the protein. These variations are mainly due to alternative splicing of the fibronectin mRNA (5).

The three types of modules are globular. Separately, their structure is known as being mainly $\beta$-sheet from X-ray and NMR studies on isolated modules $(6-8)$. However, the global conformation of fibronectin cannot be assumed from the folding of the individual isolated modules and, until now, no success in the crystallization of the whole protein has been reported. Thus, to understand the relationship between fibronectin structure and its various adhesive biological functions, a lot of studies have been performed in solution.

On the overall protein, fluorescence studies show the existence of hydrophobic domains $(9,10)$. Circular dichroism and infrared spectroscopy studies have only detected $\beta$-sheet secondary structures $(10-12)$. These results suggest that the modules within the overall protein retain the same structure as isolated.

In the absence of X-ray or NMR experimental data about the whole fibronectin, little is known about its threedimensional structure. The molecular shape of fibronectin in solution has been extensively investigated by several techniques, but its statistical conformation still remains debated (13).

The early studies of fibronectin conformation are based either (1) on a near macroscopic length scale investigation of hydrodynamic properties $(9,10,14-16)$ or static light scattering properties (17) or (2) on a more local length scale investigation using circular dichroism or fluorescence spectroscopy $(9,10,18)$.

The main result of these works is that the conformation of fibronectin in physiological conditions would imply both 
large length scale flexibility and local order. The emerging picture is that of a "string of beads". However, such a conformation determination is indirect and has been widely controversial. Thus, several authors have used more appropriate techniques to directly access to this conformation either (1) in the reciprocal space by small-angle X-ray or neutron scattering (19) or (2) in the direct space by microscopy techniques (20-24).

The above results are quite contradictory. Using scattering techniques, a compact and globular conformation is reported in physiological conditions. On the opposite, microscopy studies mainly show a "string of beads"-like conformation but also either a globular or an extended straight strand conformation depending on sample preparation.

In addition to the above suggestions, from fluorescence polarization experiments, another structural model consisting of two independently rotating disk-shaped subunits has even been proposed (25). These contradictory suggestions show the difficulty to validate a model on the basis of a unique technique. Principally, the difficulty follows from the probable complex fibronectin conformation. To summarize, the common opinion for the fibronectin conformation in physiological conditions is in favor of the string of beads model. However, up to now, X-ray and neutron-scattering experiments seem to disagree.

This paper deals with the light-scattering (elastic and quasielastic) and small-angle neutron-scattering study of fibronectin conformation in dilute solution. For a better understanding, results obtained in "native" conditions ${ }^{2}$ are compared to those obtained in the presence of denaturing agent (urea $8 \mathrm{M})$ and to theoretical expectations for globular and ideal polymer coil conformations (Gaussian statistics). In "native" conditions, our data show that fibronectin conformation varies according to the observation length scale: the chain is Gaussian at large scales and globular at smaller ones. This pleads unambiguously in favor of the string of beads model already reported using other techniques. In the presence of denaturing agents, the global conformation turns to an excluded volume chain.

The results allow the discussion of the fibronectin conformation in terms of its structural and biological implications as far as ECM organization is concerned.

\section{MATERIALS AND METHODS}

Fibronectin Purification. Fibronectin was purified from human cryoprecipitated plasma according to a new protocol developed in our laboratory that yields high quantity and purity protein (26). This protocol is based on fibronectin affinities for both gelatin and heparin. Briefly, it consists of three liquid chromatography steps: a gelatin affinity, followed by a heparin affinity, and, finally, another gelatinaffinity chromatography. All chromatography gels were purchased from Chisso Corp., Japan. The purity of the preparation was determined by densitometry analysis of silver nitrate-stained SDS - polyacrylamide gel electrophoresis, to $(98.6 \pm 1.2) \%(w / w)$. Fibronectin solution in $25 \mathrm{mM}, \mathrm{pH}$ 7.4, Tris $\mathrm{HCl}$ buffer, containing $0.5 \mathrm{mM}$ EDTA, was

\footnotetext{
${ }^{2}$ In this paper, the term "native condition" is used for conciseness. It means that the protein is prepared in nondenaturing conditions and is in solution at $\mathrm{pH} 7.4$ and $[\mathrm{NaCl}] 150 \mathrm{mM}$. These conditions correspond to $\mathrm{pH}$ and ionic strength of physiological plasma.
}

concentrated just before use, using Ultrafree-15 centrifugal concentrators from Millipore (100 kg/mol cut off). Fibronectin concentrations were determined by optical absorbance measurements: $A_{280 \mathrm{~nm}}^{1 \%}=12.8(27)$.

Samples Preparation. Fibronectin solution, $1 \mathrm{~cm}^{3}$, at concentration of the order of $10 \mathrm{mg} / \mathrm{cm}^{3}$, was dialyzed $(2 \times$ $24 \mathrm{~h}$, at room temperature) using a micro dialysis cassette (Slide-A-Lyzer, 10000 MW cut off, Pierce, Rockford, IL) against large volumes $\left(\geq 100 \mathrm{~cm}^{3}\right)$ of solution containing 25 $\mathrm{mM}$ Tris- $\mathrm{HCl}$ (pH 7.4), $0.5 \mathrm{mM}$ EDTA, and $150 \mathrm{mM} \mathrm{NaCl}{ }^{2}$ For experiments on denatured protein, $8 \mathrm{M}$ urea was added to dialysis bath. For neutron-scattering experiments, these dialyzes were realized using deuterated compounds (water, Tris, Urea, and DCl, purchased from Eurisotop, Saclay, France).

Scattering Experiments. (1) Fundamentals of Elastic Light Scattering and Small-Angle Neutron Scattering. The basic principles (28) of elastic light scattering and small-angle neutron scattering are essentially identical. An incident plane wave of wavelength $\lambda_{0}$ is scattered by the elementary scatterers (monomers of a macromolecule, small solvent molecules, ...). The interference pattern is measured as a function of the scattering vector $q=4 \pi / \lambda \times \sin (\theta / 2)$ with $\theta$ the scattering angle and $\lambda$ the actual wavelength in the medium (for light scattering $\lambda=\lambda_{0} / n$ with $n$ the refractive index of the sample). For macromolecules in solution, after data treatment (29) one accesses to the quantity $\tilde{\Sigma}(q)$, which only reflects the macromolecules contribution to the scattered intensity. For neutronicians, $\tilde{\Sigma}(q)$ is the "coherent scattering cross-section per unit volume" usually named "excess Rayleigh ratio" by light-scattering experimentalists. Very generally, one has

$$
\tilde{\Sigma}(q)=K^{2} C M S(q)
$$

where $\tilde{\Sigma}(q)$ is expressed in centimeters ${ }^{-1} ; C\left(\mathrm{~g} / \mathrm{cm}^{3}\right)$ is the concentration; $M(\mathrm{~g} / \mathrm{mol})$ the molecular mass of the macromolecules in solution; $K^{2}\left(\mathrm{~mol} \mathrm{~cm} \mathrm{~g} \mathrm{~g}^{-2}\right)$ is the contrast factor. The scattering function $S(q)$ expresses the interference of waves scattered by monomers belonging to the same macromolecule but also to different macromolecules. The intramolecular term $P(q)$ is the form factor of the macromolecules: $P(q)=\left(1 / N^{2}\right) \sum_{n=1}^{N} \sum_{m=1}^{N}\left\langle e^{i q\left(r_{m}-r_{m}\right)}\right\rangle, N$ being the number of monomers of the macromolecule and $r$ their position vectors. The brackets mean an average over all the possible conformations. For free rotating macromolecules, an average over all orientations leads to

$$
P(q)=\frac{1}{N^{2}} \sum_{n=1}^{N} \sum_{m=1}^{N}\left\langle\frac{\sin \left(q r_{n, m}\right)}{q r_{n, m}}\right\rangle
$$

with $r_{n, m}=\left|\mathbf{r}_{n}-\mathbf{r}_{m}\right|$. At small $q\left(q r_{n, m}<1\right)$, one obtains the Guinier formula $P(q)=1-q^{2} R_{\mathrm{g}}{ }^{2} / 3+\ldots$, where $R_{\mathrm{g}}{ }^{2}=(1 /$ $\left.2 N^{2}\right) \sum_{n=1}^{N} \sum_{m=1}^{N}\left\langle r_{n, m}^{2}\right\rangle$ is the averaged square radius of gyration of the macromolecule. In the Guinier $q$-range $\left(q R_{\mathrm{g}}<\right.$ 1 ), due to the average over all the orientations, the macromolecules are viewed as spherically symmetrical. In that case, the scattering function $S(q)$ can be factorized into the product of $P(q)$ and of the structure factor $G(q)$. This last factor is directly linked to the osmotic compressibility $k T(C / M)[C(\mathrm{~d} \pi /$ $\mathrm{d} C)]^{-1}$, with $\pi$ the osmotic pressure of the solution and $k T$ 
the thermal energy. For macromolecules in weak interaction, a virial expansion of the osmotic pressure can be used: $\pi=$ $\pi_{\mathrm{c} \rightarrow 0}\left(1+\mathrm{MA}_{2} C+\ldots\right)$, with $\mathrm{MA}_{2}$ the two bodies interaction term. Finally,

$$
\begin{aligned}
& \frac{\tilde{\Sigma}(q)_{q R_{\mathrm{g}}<1, \mathrm{MA}_{2} C}<1}{C}= \\
& K^{2} M\left(1-\frac{\mathrm{q}^{2} R_{\mathrm{g}}{ }^{2}}{3}+\ldots\right)\left(1-2 \mathrm{MA}_{2} C+\ldots\right)
\end{aligned}
$$

At zero $q$, elastic-scattering experiments are sensitive to the osmotic compressibility of the solution, i.e., a macroscopic property. In this limit, the macromolecules are pointlike. For increasing $q$, the size of the macromolecules is beginning to emerge, allowing the radius of gyration to be determined. Actually, the scattering vector $q$ acts as the magnifying of a microscope. For even higher $q$ value $\left(q R_{\mathrm{g}}\right.$ $>1$ in dilute solution), the intermolecular interference term can be neglected and scattering experiments are only sensitive to the form factor $P(q)$, reflecting the internal conformation of a single macromolecule.

Exact calculation of the form factor can be performed only in a few cases. For instance, a sphere of radius $R$ gives $P(q)$ $=\left[3 \times(\sin (q R)-q R \cos (q R)) /(q R)^{3}\right]^{2}$, with characteristic oscillations at $q R>1$, which come down to a strong $q^{-4}$ dependence of the scattered intensity using low-resolution spectrometers (as small angle neutron scattering spectrometers). Note that such an oscillation smoothing can also be due to a size distribution of the spheres. Finally, the sphere form factor can be viewed as a reference and generic behavior for dense and compact structures, the $q^{-4}$ behavior being the signature of a sharp interface between the inner and outer parts of the macromolecule. Another borderline case, which is valuable to consider for comparison with experiment, is the ideal polymer chain obeying to Gaussian statistics. Ideal chain corresponds to a random walk of $N$ steps of length $b$. Its radius of gyration $R_{\mathrm{g}}$ is known as being equal to $N b^{2} / 6$. For observation length scales $q^{-1}$ large enough compared to $b$, the form factor of the ideal chain obeys to the Debye function $P(q)=\left(2 / x^{2}\right)\left(\mathrm{e}^{-x}-1+x\right)$ with $x=q^{2} R_{\mathrm{g}}^{2}$. At $q R_{\mathrm{g}}>1$, the Debye function displays a characteristic $q^{-2}$ dependence reflecting the random walk fractal dimension equal to 2. However, in good solvent condition, the polymer is swelled due to excluded volume interactions. In that case, the chain conformation rather corresponds to a self-avoiding random walk leading to a $q^{-1.7}$ dependence of the form factor.

(2) Quasi-Elastic Light Scattering. Concentration fluctuations within the scattering volume result in scattered intensity fluctuations. Thus, computing the time-dependent correlation function of the scattered intensity (30) yields the timedependent structure factor $S(q, t)$. At large length scales $q^{-1}$, compared to the radius of gyration $R_{\mathrm{g}}$ of macromolecules, only their translational motion contributes to concentration fluctuations. If only one species of macromolecule is in solution, the relaxation function of concentration fluctuations is a single exponential with a characteristic time corresponding to that needed for a macromolecule to cover a $q^{-2}$ area through Brownian motion. $S(q, t)$ can thus be written as $S(q, t)$ $=\mathrm{e}^{-D q^{2} t}$, with $D$ the diffusion coefficient of the macromolecules. According to the Stockes-Einstein relation, the diffusion coefficient is inversely proportional to the hydrodynamic radius $R_{\mathrm{H}}$, i.e., the radius of the sphere which would encounter the same friction, $R_{\mathrm{H}}=k T / 6 \pi \eta D$. For spheres, e.g., polymers having a globular and compact structure, $R_{\mathrm{H}}$ is equal to the radius of the sphere $\sqrt{5 / 3} R_{g}$. However, for polymer chains in the Zimm limit of hydrodynamic interactions between monomers, $R_{\mathrm{H}}$ is an average value, the calculation (31) of which differing from that of $R_{\mathrm{g}}$. Actually, $1 / R_{\mathrm{H}}=\left(1 / 2 N^{2}\right) \sum_{n=1}^{N} \sum_{m=1}^{N}\left\langle 1 / r_{n, m}\right\rangle$. The different way of averaging for $R_{\mathrm{g}}$ and $R_{\mathrm{H}}$ is the source of different integration constants for both radii leading to a ratio $R_{\mathrm{H}} / R_{\mathrm{g}}$ bearing information on the chain statistical conformation. For instance, $R_{\mathrm{H}} / R_{\mathrm{g}}=0.80$ for an ideal Gaussian chain and $R_{\mathrm{H}} /$ $R_{\mathrm{g}}=0.64$ for excluded volume chain (32). However, these values correspond to asymptotical behaviors that are observed for sufficiently long chains, whereas for shorter chains, smaller ratios are found experimentally and explained theoretically (33).

(3) Experiment Setup and Measurement Conditions. Lightscattering experiments were performed using vertically polarized Argon gas laser of wavelength $\lambda_{0}=488 \mathrm{~nm}$. Scattered intensity is measured in the horizontal plane at different scattering angle from 20 to $150^{\circ}$. The total scattered intensity per scattering volume unit of toluene taken as reference is measured to be independent of the scattering angle within $1 \%$ error bars. The Rayleigh ratio of toluene is equal to $R$ toluene $=42.7 \times 10^{-6} \mathrm{~cm}^{-1}$. The refractive index $n_{0}$ of urea solutions was measured using an Abbe refratometer: $n_{0}=1.333 \times\left(1+6.52 \times 10^{-3}\right.$ [urea] $)$, with [urea] in moles per liter. The refractive index increment $\mathrm{d} n / \mathrm{d} C$ of fibronectin cannot be easily measured in our case because these measurements should be performed in a concentrated regime and thus should require a large amount of protein. From literature, the refractive index increment of proteins in water is $(\mathrm{d} n / \mathrm{d} C)_{\text {water }}=0.200 \mathrm{~cm}^{3} \mathrm{~g}^{-1}$ at $\lambda_{0}=488 \mathrm{~nm}$. In the case of proteins in urea solutions, no value of $\mathrm{d} n / \mathrm{d} C$ is available to our knowledge. Thus, we have calculated the refractive index increment following the equation

$$
\left(\frac{\mathrm{d} n}{\mathrm{~d} C}\right)_{8 \text { Murea }}=\frac{\rho\left(\frac{\mathrm{d} n}{\mathrm{~d} C}\right)_{\text {water }}-\Delta n_{8 \text { Murea/water }}}{\rho}
$$

where $\rho=1.39 \mathrm{~g} / \mathrm{cm}^{3}$ is the fibronectin density (9) and $\Delta n_{8 \mathrm{Murea} / \mathrm{water}}=0.067$ is the difference between the refractive index of an $8 \mathrm{M}$ urea solution and pure water. Such equation is based on a mixing law argument. It gives $(\mathrm{d} n / \mathrm{d} C)_{8 \text { Murea }}=$ $0.152 \mathrm{~cm}^{3} \mathrm{~g}^{-1}$. The light-scattering contrast factor

$$
K^{2}=\left(\frac{2 \pi n_{0}}{\lambda_{0}^{2} N_{\mathrm{a}}} \times \frac{\mathrm{d} n}{\mathrm{~d} C}\right)^{2}
$$

where $N_{\mathrm{a}}$ is the Avogadro's number, was thus calculated as equal to $8.22 \times 10^{-7} \mathrm{~cm}^{2} \mathrm{~g}^{-2} \mathrm{~mol}$ for protein in water and to $5.23 \times 10^{-7} \mathrm{~cm}^{2} \mathrm{~g}^{-2} \mathrm{~mol}$ for protein in $8 \mathrm{M}$ urea solutions. Quasi-elastic light-scattering measurements were performed computing the time-dependent autocorrelation function of the scattered intensity with a Malvern 7032 correlator. The viscosity $\eta$ of urea solutions was measured at $20^{\circ} \mathrm{C}$ using an Ubbelohde capillary viscometer: $\eta(\mathrm{cp})=1.0309 \times(1$ +0.0475 [urea]). 
Small-angle neutron-scattering measurements were performed with the PACE spectrometer at the Laboratoire Léon Brillouin. To cover the widest $q$-range, three configurations (distance from sample to multidetector/wavelength) were used: $4.7 \mathrm{~m} / 15 \AA, 2.3 \mathrm{~m} / 5 \AA$, and $0.6 \mathrm{~m} / 4 \AA$. Initial data treatment was carried out following Cotton (29). The contrast factor $K^{2}$ was calculated following Jacrot (34). For fibronectin in $\mathrm{D}_{2} \mathrm{O}, K^{2}=1.10 \times 10^{-3} \mathrm{~cm}^{2} \mathrm{~g}^{-2}$ mol assuming that $80 \%$ of labile hydrogens is actually exchanged (35). For fibronectin in $\mathrm{D}_{2} \mathrm{O} / 8 \mathrm{M}$ urea, $K^{2}=1.22 \times 10^{-3} \mathrm{~cm}^{2} \mathrm{~g}^{-2} \mathrm{~mol}$ assuming that all labile hydrogens are exchanged.

\section{RESULTS}

Scattered Intensity at $q \rightarrow 0$. Light-scattering experiments were performed on fibronectin solutions in the presence of salt $(150 \mathrm{mM} \mathrm{NaCl})$ for protein concentration $C$ between 0.2 and $10 \mathrm{mg} / \mathrm{cm}^{3}$. The $q$-dependent scattered intensity was analyzed using eq 3 . The values of radii of gyration are discussed below. Extrapolation to zero $q$ of the scattering cross-section leads to the apparent molecular mass of the protein in solution: $M_{\text {app }}=\tilde{\Sigma}(q \rightarrow 0, C) / K^{2} C=M \times(1-$ $\left.2 \mathrm{MA}_{2} C\right)$. Results are plotted in Figure 1, for fibronectin in "native" conditions and in the presence of $8 \mathrm{M}$ urea, respectively. First, note that error bars are much more important for measurements performed in "native" condition than for $8 \mathrm{M}$ urea solutions. In the former case, the very small $q$ dependence (small radius of gyration) of the scattered intensity is responsible for the inaccuracy of the zero $q$ extrapolation. However, the apparent molecular mass $M_{\text {app }}$ is found to be concentration independent in "native" conditions, i.e., the second virial is unmeasurable and near zero. In presence of $8 \mathrm{M}$ urea, the apparent molecular mass $M_{\text {app }}$ is strongly sensitive to concentration. The second virial coefficient deduced from the slope of $M_{\text {app }}$ vs $C$ is equal to

$$
\mathrm{MA}_{2,8 \text { Murea }}=(58 \pm 5) \mathrm{cm}^{3} / \mathrm{g}
$$

In terms of concentration, $1 / \mathrm{MA}_{2}$ is the concentration at which proteins strongly interact and are not any more in a dilute regime. This concentration is equal to $1 / \mathrm{MA}_{2}=17$ $\mathrm{mg} / \mathrm{cm}^{3}$.

Extrapolation to zero concentration of the apparent molecular mass leads to the actual molecular mass of fibronectin deduced from our measurements. One obtains $M=(570 \pm$ $50) \mathrm{kg} / \mathrm{mol}$ in "native" conditions and $M=(590 \pm 30) \mathrm{kg} /$ mol in $8 \mathrm{M}$ urea. These measurements are in rather good agreement with the value $530 \mathrm{~kg} / \mathrm{mol}$ calculated from the fibronectin primary structure. This calculated value is actually increased by the glycosylation that is of the order of $4 \%$ of the molecular mass (1). The value measured with urea is slightly higher than the expected molecular mass, but this can be imputed to our calculation of refractive index increment.

Radius of Gyration. In the Guinier range $\left(\mathrm{q} R_{\mathrm{g}}<1\right)$, the radius of gyration $R_{\mathrm{g}}$ of fibronectin can be determined from the $q$ dependence of the scattered intensity. In the case of "native" fibronectin, $R_{\mathrm{g}}$ is found to have a small value with respect to the light-scattering $q$-range, leading to a weak $q$ dependence of light-scattered intensity (see eq 3 ). On the contrary, $R_{\mathrm{g}}$ is found to have a high value with respect to small-angle neutron-scattering $q$-range, leading to a reduced Guinier range. However, the use of both techniques gives a

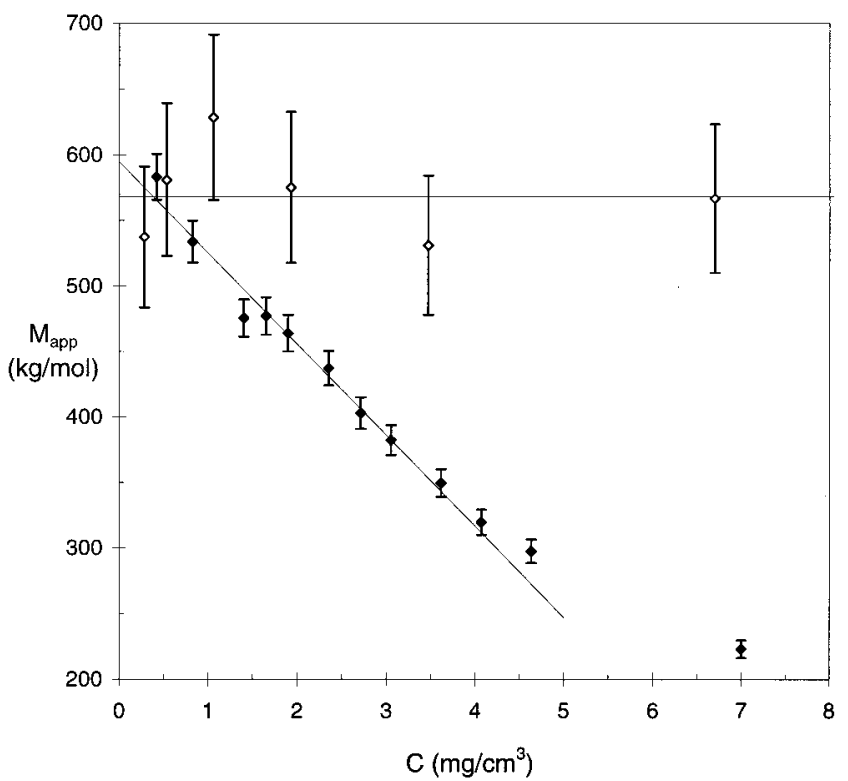

FIGURE 1: Apparent molecular mass $M_{\text {app }}=\tilde{\Sigma}(q \rightarrow 0, C) / K^{2} C$, deduced from light scattered intensity measurements, vs concentration, $C$. In "native" conditions $(\diamond), M_{\text {app }}$ is constant within experimental accuracy, i.e., the second virial coefficient $\left(\mathrm{MA}_{2}\right)$ is unmeasurable. In $8 \mathrm{M}$ urea solution $(\diamond)$, the second virial coefficient deduced from the slope of the straight line is equal to $\mathrm{MA}_{2,8} \mathrm{M}$ urea $=(58 \pm 5) \mathrm{cm}^{3} / \mathrm{g}$.

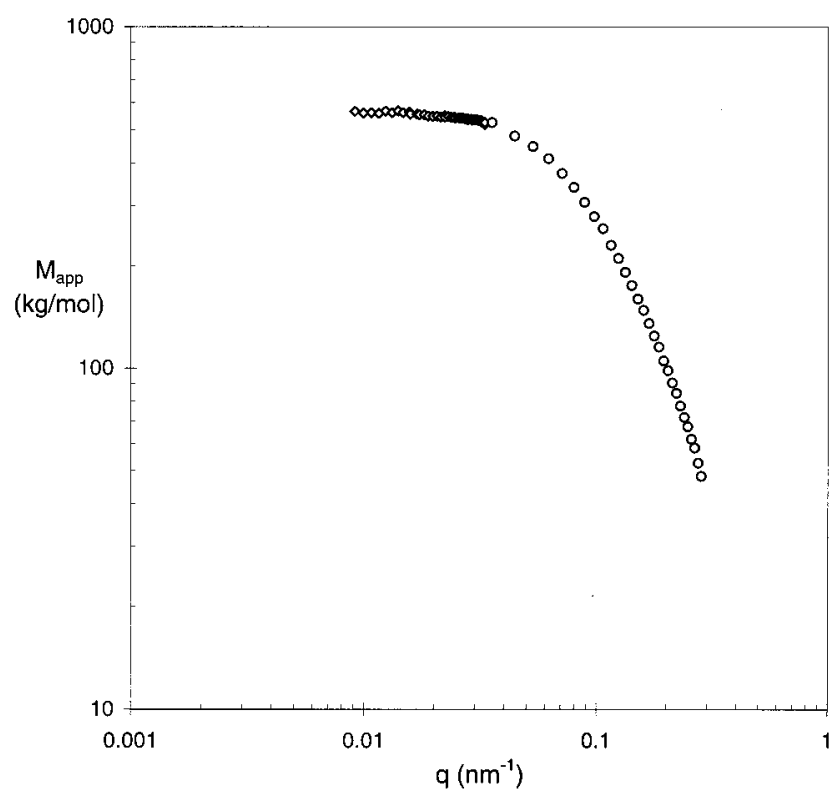

FIGURE 2: Scattering cross section per concentration unit and contrast unit, $M_{\text {app }}(q)=\tilde{\Sigma}(q) / K^{2} C$, as a function of scattering vector $\mathrm{q}$, for fibronectin in "native" condition. Diamonds correspond to light scattering measurements and circles to small-angle neutron scattering measurements. At $q \longrightarrow 0, M_{\text {app }}$ is equal to the molecular mass of fibronectin.

good estimate of $R_{\mathrm{g}}$. In Figure 2, the scattering cross-section per concentration unit, $M_{\text {app }}(q)=\tilde{\Sigma}(q) / K^{2} C$, is plotted as a function of the scattering vector $q$. This figure contains both results obtained by light scattering (at a concentration $C=$ $\left.6.7 \times 10^{-3} \mathrm{~g} / \mathrm{cm}^{3}\right)$ and neutron scattering $\left(C=7.1 \times 10^{-3}\right.$ $\left.\mathrm{g} / \mathrm{cm}^{3}\right)$. The data are expressed in units $(\mathrm{g} / \mathrm{mol})$ accounting for the different contrast factor of both techniques. In the overlapping $q$-range of both measurements, one notes a fair superposition of data, making us confident in the measurements performed. Classically, the curvature of the scattering 


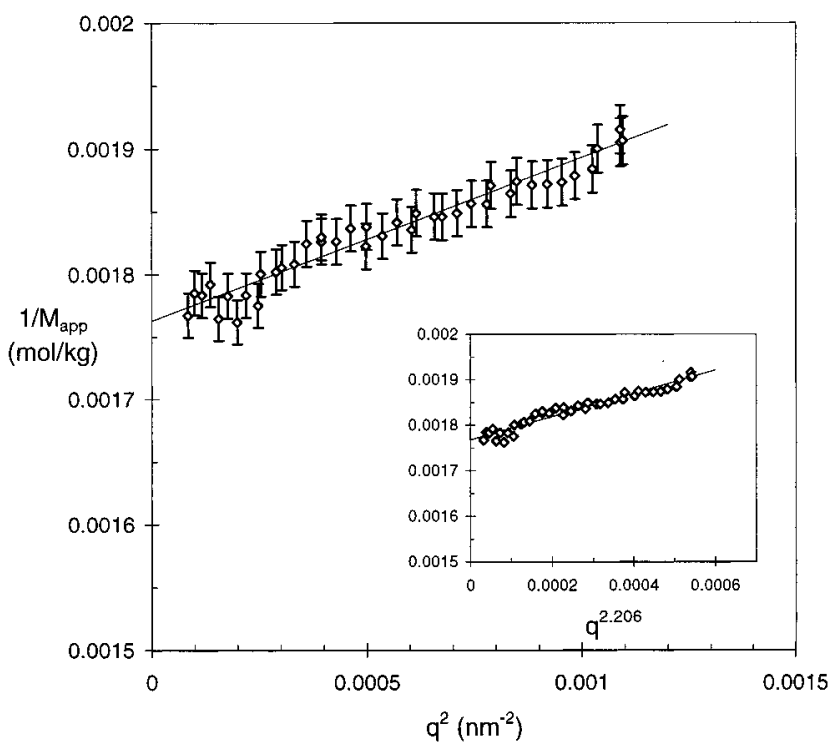

FIGURE 3: Reverse scattering cross-section per concentration unit and contrast unit, $1 / M_{\text {app }}(q)=K^{2} C / \tilde{\Sigma}(q)$ vs $q^{2}$ (Zimm approximation) and vs $q^{2.206}$ (see inset, Calmettes' approximation). Measurements performed by light scattering for fibronectin in "native" condition at a concentration $C=6.7 \mathrm{mg} / \mathrm{cm}^{3}$. Straight lines correspond to linear fits. Both approximations leads to the same radius of gyration $R_{\mathrm{g}}=(15.1 \pm 0.1) \mathrm{nm}$ and to the molecular mass $M_{\text {app }, q \rightarrow 0}=(570$ $\pm 10) \mathrm{kg} / \mathrm{mol}$.

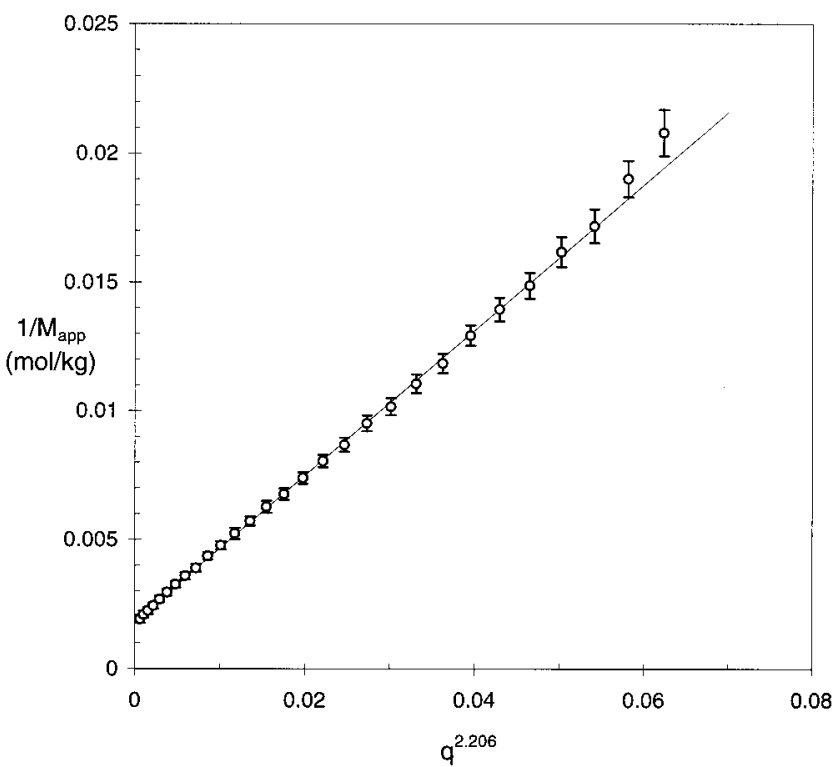

FIGURE 4: Reverse scattering cross-section per concentration unit and contrast unit, $1 / M_{\text {app }}(q)=K^{2} C / \tilde{\Sigma}(q)$, measured by small angle neutron scattering for fibronectin in "native" condition at a concentration $C=14 \mathrm{mg} / \mathrm{cm}^{3}$, as a function of $q^{2.206}$ (Calmettes' approximation). Straight line corresponds to a linear fit leading to the same radius of gyration $R_{\mathrm{g}}=(15.6 \pm 0.2) \mathrm{nm}$ as that measured by light scattering and to the molecular mass $M_{\text {app, } q \rightarrow 0}=(545 \pm$ 10) $\mathrm{kg} / \mathrm{mol}$.

profile corresponds to scattering vectors of the order of $1 / R_{\mathrm{g}}$. To have a more accurate determination of $R_{\mathrm{g}}$, light-scattering data have been fitted using the Zimm approximation, $\tilde{\Sigma}_{q \rightarrow 0} / \tilde{\Sigma}$ $=\left[1+\left(q R_{\mathrm{g}}\right)^{2} / 3\right]$ (Figure 3), and the Calmettes' approximation (36), $\tilde{\Sigma}_{q \rightarrow 0} / \tilde{\Sigma}=\left[1+0.359\left(q R_{\mathrm{g}}\right)^{2.206 / 3}\right]$ (Figure 3, inset), which is known to linearize the Debye function up to $q R_{\mathrm{g}} \cong$ 4. The two approximations lead to the same radius of gyration within error bars: $R_{\mathrm{g}}=(15.1 \pm 0.1) \mathrm{nm}$. The above

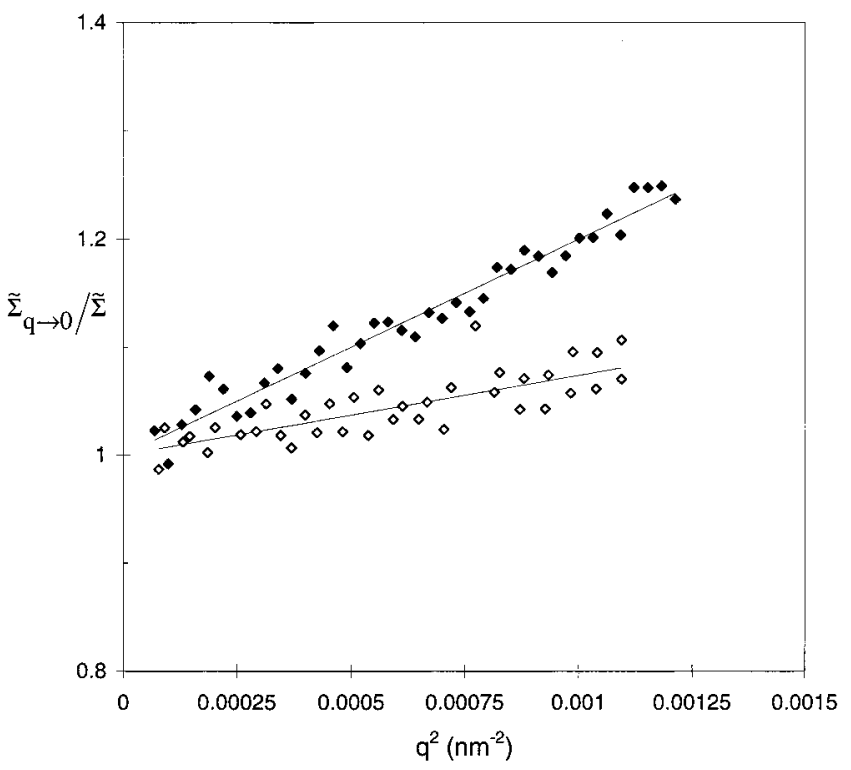

FIGURE 5: Normalized inverse light scattered intensity $\tilde{\Sigma}_{q \rightarrow 0} / \tilde{\Sigma}$ as a function $q^{2}$ for "native" fibronectin in "native" conditions $(\diamond)$ and in a presence $8 \mathrm{M}$ urea $(\diamond)$. Measurement performed at concentration $C=7.1 \mathrm{mg} / \mathrm{cm}^{3}$ for "native" protein and in a more dilute solution $\left(C=1.46 \mathrm{mg} / \mathrm{cm}^{3}\right)$ for denatured protein because of the higher second virial coefficient. Straight lines correspond to the corresponding Zimm approximation fits: $\tilde{\Sigma}_{q \rightarrow 0} / \tilde{\Sigma}=1+q^{2} \times$ $R_{\mathrm{g}}{ }^{2} / 3$.

value of $R_{\mathrm{g}}$ reduces so much the Guinier range that it is not possible to fit small-angle neutron-scattering data using the Zimm approximation with the constraint $q R_{\mathrm{g}}<1$. However, the $1 / \tilde{\Sigma}$ vs $q^{2.206}$ representation allows us to fit the data (see Figure 4) yielding $R_{\mathrm{g}}=(15.6 \pm 0.2) \mathrm{nm}$. The values determined using the two scattering techniques are in good agreement. In addition, no systematic dependence of $R_{\mathrm{g}}$ on concentration has been brought out, within error bars in the concentration range here studied $\left(C<15 \mathrm{mg} / \mathrm{cm}^{3}\right)$. Finally, the mean value is

$$
R_{\mathrm{g}, \text { "native" }}=(15.3 \pm 0.2) \mathrm{nm}
$$

In presence of $8 \mathrm{M}$ urea, an increase of the radius of gyration is observed. In Figure 5, the normalized inverse light-scattered intensity $\tilde{\Sigma}_{q \rightarrow 0} / \tilde{\Sigma}$ as a function of $q^{2}$ is plotted and compared to measurements obtained for the "native" protein. The increasing slope in the presence of urea gives evidence for the increase of $R_{\mathrm{g}}$. In $8 \mathrm{M}$ urea solutions, the radius is too high to be measured with small-angle neutron scattering. The value measured by light scattering is concentration dependent, i.e., sensitive on protein-protein interactions. In Figure 6, the measured values are plotted as a function of protein concentration. The linear behavior allows us to extrapolate the apparent $R_{\mathrm{g}}$ values to zero concentration, leading to the actual size of fibronectin in 8 M urea:

$$
R_{\mathrm{g}, 8 \text { Murea }}=(30 \pm 1) \mathrm{nm}
$$

Quasi-Elastic Light Scattering. Quasi-elastic light-scattering measurements were performed in "native" solutions and $8 \mathrm{M}$ urea solution. In both conditions, the dynamical structure factor measured at different scattering angles can be rescaled using $t q^{2}$ as variable. Without any data fit, this 


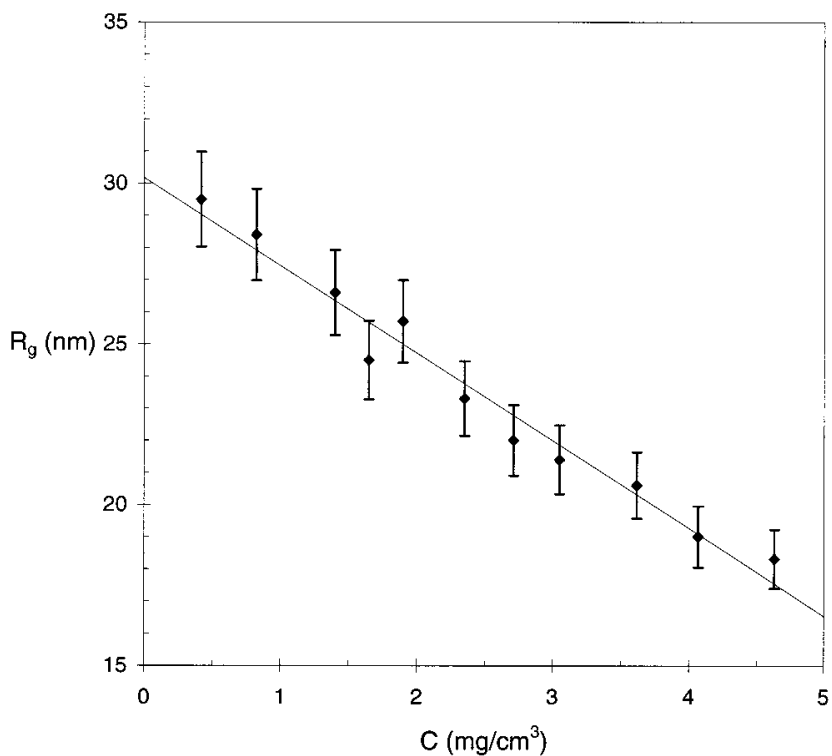

FIGURE 6: Apparent radius of gyration $R_{\mathrm{g}}$ as a function of protein concentration, $C$, for fibronectin solutions in the presence of $8 \mathrm{M}$ urea $(150 \mathrm{mM} \mathrm{NaCl})$. The straight line is a linear fit leading to the extrapolated value $R_{\mathrm{g}, 8 \mathrm{M} \text { urea }}=(30 \pm 1) \mathrm{nm}$ at zero concentration.

gives evidence that a diffusion process is probed. In Figure 7, $S(q, t)$ is plotted as a function of $t q^{2} / \eta$. The time axis is divided by the solvent viscosity $\eta$ in order to get free of trivial effects due to the different viscosities of the two solvents. In log-linear scale, the linear behavior of $S(q, t)$ clearly indicates the simple exponential relaxation ensuring that no aggregation takes place. Thus, in this representation, the slope is equal to $D \eta=k T / 6 \pi R_{\mathrm{H}}$, with $D$ the diffusion coefficient and $R_{\mathrm{H}}$ the hydrodynamic radius of fibronectin differing in "native" conditions and $8 \mathrm{M}$ urea solution (see inset of Figure 7). The averaged values of the hydrodynamic radius are

$$
\begin{aligned}
R_{\mathrm{H}, \text { "native" }} & =(11.5 \pm 0.1) \mathrm{nm} \\
R_{\mathrm{H}, 8 \mathrm{M} \text {-urea }} & =(18.3 \pm 0.3) \mathrm{nm}
\end{aligned}
$$

In both conditions, no significant concentration effect was observed on measurements. This can be explained by a wellknown cancellation of hydrodynamic and thermodynamic interactions that operate with opposite signs in the expression of the diffusion coefficient (31).

Small-Angle Neutron Scattering. To probe the internal conformation of fibronectin, the neutron-scattering intensity of fibronectin solutions was collected in a wide $q$-range corresponding to length scales: $0.2<q^{-1}(\mathrm{~nm})<30$. In this $q$-range and in dilute solution, the intermolecular contribution to the scattering function can be neglected, and the normalized scattering cross-section $\tilde{\Sigma}(q) / \tilde{\Sigma}_{q \rightarrow 0}$ gives a good approximation of the form factor. In Figure $8, \tilde{\Sigma}(q) /$ $\tilde{\Sigma}_{q \rightarrow 0}$ is plotted as a function of $q R_{\mathrm{g}}$ for "native" and denatured protein, respectively. At zero $q$, the two curves tend to one because of normalization. Such normalization is needed for the comparison of the curves. Actually, accounting for the contrast factors (which differ with and without urea) and for the concentration, the two curves should join the value corresponding to the molecular mass of fibronectin, which remains unchanged with urea addition. However, due to the

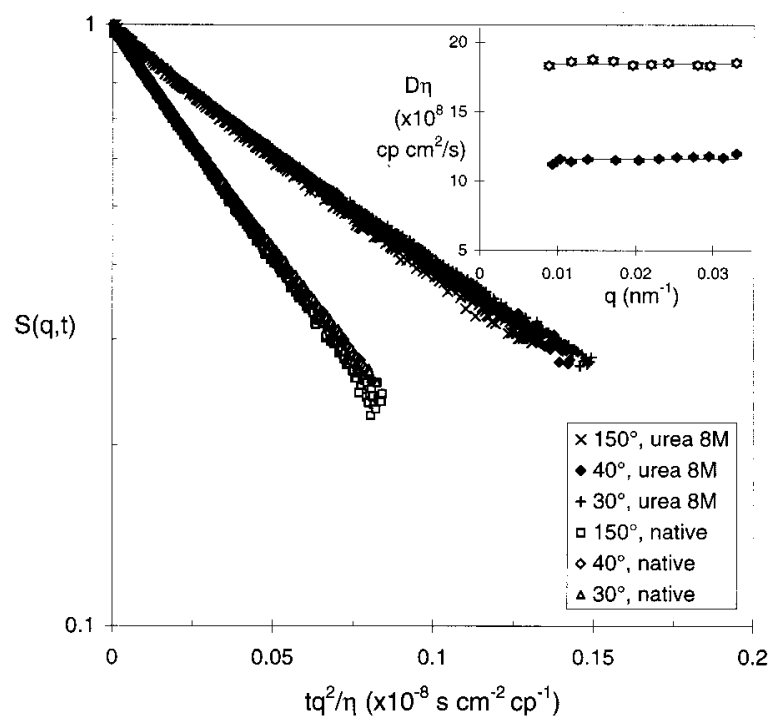

FIGURE 7: Dynamical structure factor $S(q, t)$ as a function of the reduced variable $t q^{2} / \eta$, where $t$ is the time, $q$ the scattering vector, and $\eta$ the solvent viscosity. Measurements performed in "native" conditions ("native" protein, $C=7.1 \mathrm{mg} / \mathrm{cm}^{3}$ ) and in $8 \mathrm{M}$ urea solution (denatured protein, $C=3.5 \mathrm{mg} / \mathrm{cm}^{3}$ ) at three different scattering angles (see legend). The slope of the curves correspond to $D \times \eta=k T / 6 \pi R_{\mathrm{H}}$, where $D$ is the diffusion coefficient and $R_{\mathrm{H}}$ the hydrodynamic radius. In the inset the product $D \times \eta$ is plotted as a function of $q$.

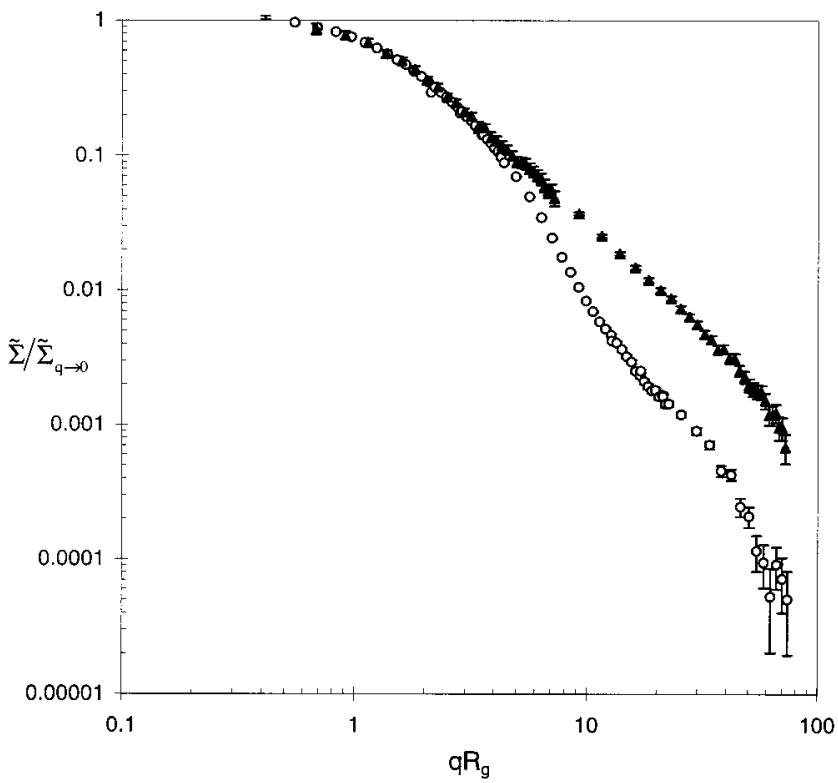

FIGURE 8: Reduced scattering cross-section $\tilde{\Sigma} / \tilde{\Sigma}_{q \rightarrow 0}$ of "native" fibronectin $\left[C=14 \mathrm{mg} / \mathrm{cm}^{3}(\mathrm{O})\right]$ and urea $8 \mathrm{M}$ denatured fibronectin $\left[C=6 \mathrm{mg} / \mathrm{cm}^{3}(\Delta)\right]$ measured by small-angle neutron scattering as a function of $q R_{\mathrm{g}}$.

second virial coefficient that is nonzero with urea, this is not quite correct, and at zero, $q \tilde{\Sigma}(q) / K^{2} C$ tends only to an apparent molecular mass. Normalization allows us to get free of such an effect. Nevertheless, the two curves differ strongly in the whole $q$-range. In $8 \mathrm{M}$ urea solution, the form factor at $q R_{\mathrm{g}}>1$ displays a power law behavior over more than one decade. The corresponding exponent is equal to 1.7 as expected for a completely unfolded protein. In "native" solution, no power law is observed; the form factor does not show the characteristics of scattering by polymers neither in coil nor in globular conformation. The form factor is much 
more complex and will be discussed in details in the next section.

\section{DISCUSSION}

To elucidate the "native" fibronectin conformation, comparison with the denatured conformation is useful. Our results give evidence for an increase of both hydrodynamic radius and radius of gyration with $8 \mathrm{M}$ urea addition, i.e., with respect to a completely unfolded conformation. The hydrodynamic radius increases by a factor of 1.6, whereas the radius of gyration increases by a factor of 2 . That means the volume occupied by a protein molecule is increased by a factor of $2^{3}$ when unfolded. To compare the different results, let us define the protein concentration inside a protein coil or protein globule as $C^{*}=M /\left({ }^{4} /{ }_{3} \pi R_{\mathrm{g}}{ }^{3}\right)$. One obtains $C^{*} \cong$ $64 \mathrm{mg} / \mathrm{cm}^{3}$ for fibronectin in "native" conditions and $C^{*} \cong$ $8.4 \mathrm{mg} / \mathrm{cm}^{3}$ for the unfolded fibronectin. For globular proteins the value $\rho=1370 \mathrm{mg} / \mathrm{cm}^{3}$ is usually reported (37) for the density. This value leads to $C^{*} \cong 2950 \mathrm{mg} / \mathrm{cm}^{3}$ for a globular protein and using our above definition of $C^{*}\left(C^{*}\right.$ and $\rho$ differ by a factor $\sqrt{5 / 3^{3}}$ ). Such a direct comparison would indicate that "native" fibronectin adopts neither an unfolded nor a globular conformation.

Examination of the ratio $R_{\mathrm{H}} / R_{\mathrm{g}}$ gives a first indication for the conformation of the protein. The value $R_{\mathrm{H}} / R_{\mathrm{g}}=0.61 \pm$ 0.03 observed for denatured fibronectin is not very far from the value of 0.64 expected for excluded volume chain. As for the value $R_{\mathrm{H}} / R_{\mathrm{g}}=0.75 \pm 0.02$ observed in "native" conditions, it indicates clearly that "native" fibronectin is certainly not globular $\left(R_{\mathrm{H}} / R_{\mathrm{g}}=1.3\right.$ for spheres $)$. Such a ratio is much more compatible with a coil structure allowing a partial solvent-free draining through its volume. The ratio $R_{\mathrm{H}} / R_{\mathrm{g}}$ is just below the asymptotic value expected for ideal infinite polymer chains and significantly higher than the value expected in the case of excluded volume chain. Actually, this result is compatible with the zero second virial coefficient measured in "native" conditions.

The results discussed above would indicate that "native" fibronectin adopts a coillike conformation but also that it is not completely unfolded. This is quite compatible with the string of beads model already reported in the literature. To check this model, the corresponding form factor has been calculated. The simplest model accounting for this conformation corresponds to an ideal linear chain made of the 56 modules of fibronectin. Assuming a spherical shape for each module, the form factor can be rigorously factorized in two terms: the form factor $P_{\mathrm{RW}}(q)$ of a random walk of 56 unit length steps and the form factor of a spherical module of radius $1 / 2$ :

$$
P(q)=P_{\mathrm{RW}}(q) \times\left[3 \times \frac{\left(\sin \left(\frac{q}{2}\right)-\frac{q}{2} \cos \left(\frac{q}{2}\right)\right)}{\left(\frac{q}{2}\right)^{3}}\right]^{2}
$$

This is equivalent for a single macromolecule to the factorization of the scattering function in a form factor and a structure factor accounting for the position of the centers of mass. Such a factorization is often used in the case of many particles problem (28) and is applied here in our case: $P_{\mathrm{RW}}(q)$ being equivalent to the structure factor of modules. Unfortunately, exact calculation of $P_{\mathrm{RW}}(q)$ cannot be achieved,

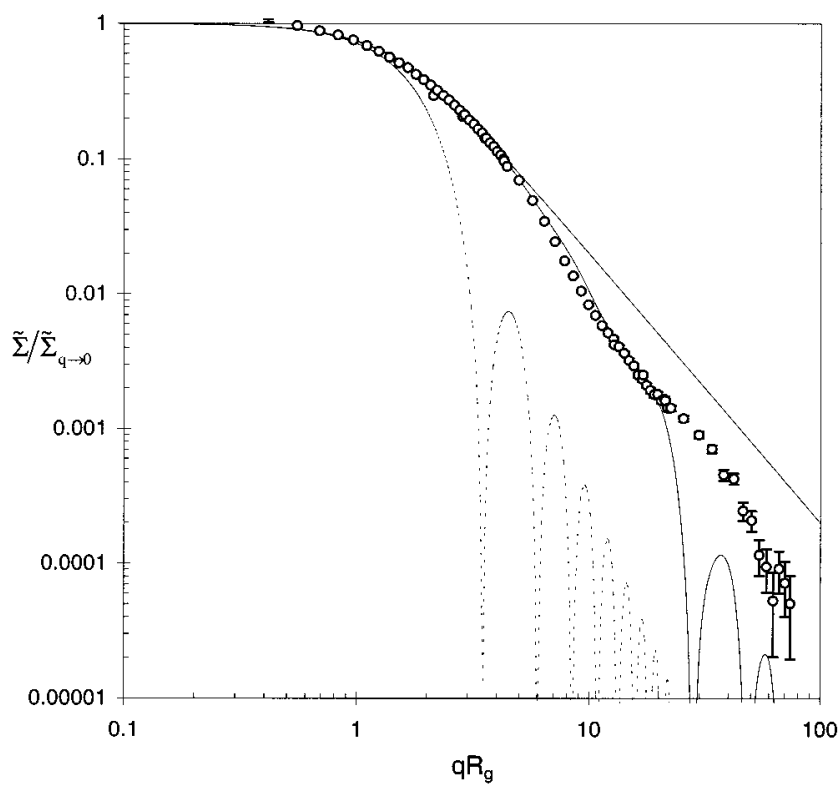

FIGURE 9: Reduced scattering cross-section $\tilde{\Sigma} / \tilde{\Sigma}_{q \rightarrow 0}$ of "native" fibronectin $\left(C=14 \mathrm{mg} / \mathrm{cm}^{3}\right)$ measured by small-angle neutron scattering as a function of $q R_{\mathrm{g}}$. Measurement is compared to the form factor of a sphere (lower curve), to the form factor of an ideal chain made of infinitely small monomers [Debye function (upper curve)], and to our calculation of the form factor for a string of 56 beads (intermediate curve).

especially due to the small number of steps leading to a radius of gyration not far from the steps length. Thus, computation of $P_{\mathrm{RW}}(q)$ was performed using a Monte Carlo method: (1) a random walk is generated; (2) its form factor is calculated and averaged over all possible orientations following eq 2 ; and (3) stages 1 and 2 are reiterated in order to average the corresponding form factor.

The result is plotted in Figure 9 and compared to smallangle neutron measurements for "native" fibronectin and theoretical expectations for sphere and Debye's function of same radius of gyration. One can see that the sphere form factor does not at all fit the data at $q R_{\mathrm{g}}>1$, whereas the Debye function accounts for the data up to $q R_{\mathrm{g}}=5$. On the other hand, the string of beads model nicely fits the data up to $q R_{\mathrm{g}} \cong 20$. It is important to note that this latter fit is obtained without any adjustable parameter.

At $q R_{\mathrm{g}}>20$, measurement is sensitive to the actual shape of the modules: (1) these modules are of three different types and sizes; (2) they are presumably not spherical but rather elliptic; and (3) they are linked together by small spacer peptide chains. All these refinements are not taken into consideration and this is the reason for the discrepancy between measurement and the model at high $q$. Accounting for point 1 would result in smoothing the oscillations of the model form factor at high $q$, in better agreement with measurement. Accounting for the elliptic shape of the module would be the most efficient modification of the model. But this is not easy to achieve because factorization of the form factor following eq 4 would not be allowed. Finally, point 3 can be easily achieved in a first approximation taking a smaller average radius of gyration for the modules. In eq 4 , this radius is taken to be equal to the half of the step length and this implies modules in contact. Finally, all these refinements would introduce at least three adjustable parameters (size distribution of modules and longitudinal and transverse radii) contrary to our model that has none. 
The validity of the string of beads model can be also checked considering the average size of the modules deduced from the model. The classical radius of gyration of a random walk leads in our case to $R_{\mathrm{g}}=b \sqrt{56 / 6}$, with $b$ the step length. Comparison with the value $R_{\mathrm{g}}=15.3 \mathrm{~nm}$ measured for the fibronectin yields $b / 2=2.5 \mathrm{~nm}$ for the average radius of modules $\left(R_{\mathrm{g} \text { module }}=(b / 2) / \sqrt{5 / 3}=1.9 \mathrm{~nm}\right)$. This is in good agreement with the overall length $b=4 \mathrm{~nm}$ of type III modules deduced from crystallography data (38).

\section{CONCLUSION}

Our main result is that fibronectin in "native" conditions has a string of beads conformation, i.e., it is globular at small length scales and obeys to Gaussian statistics at larger ones. This statistical conformation is the starting point to discuss biological implications as far as ECM organization is concerned. The ECM is a gellike network of various proteins, i.e., a network resulting from weak or covalent bonds between the chains, fibronectin being the major functional protein. Its functional properties result from its affinities for a number of biological macromolecules. For instance, in vivo, fibronectin mediates cell adhesion to the ECM and regulates cell proliferation and differentiation (1). Clearly, the fibronectin Gaussian conformation at large length scales makes easier the access of amino acids all along the flexible chain for other ligands. Moreover, the number of possible conformations of a Gaussian chain and their fluctuations are much more important compared to a globular conformation. This leads to a better adaptability for associations. At the same time, fibronectin is stable, with respect to proteinase hydrolysis, because of its globular conformation at small length scales. The ability to fulfill these functions results from its particular statistical conformation: fibronectin is not globular, unlike most intracellular or body fluid proteins. However, besides the helical collagens, many ECM proteins present modular structures composed of repeated globular domains, similar to that of fibronectin (this is the case for vitronectin, laminin, and tenascin). The "string of beads" conformation could thus correspond to a widespread protein conformation in the ECM.

In this paper, the fibronectin conformation was studied in dilute solution at $\mathrm{pH}$ and ionic strength corresponding roughly to that of blood plasma. Note that, in the ECM, fibronectin concentration is presumably higher, allowing fibronectin self-association. In addition, fibronectin associates with many other ECM constituents, most of them being less flexible than fibronectin. Finally, in the ECM, the ionic strength is presumably higher than in the plasma because of the high polyelectrolyte concentration. The conformation of fibronectin in the ECM is probably affected by these different physicochemical conditions. Investigations in these conditions would be fruitful for biological understanding of the ECM organization and function.

Finally, note that no crystallization of the whole fibronectin has been reported in the literature. At first sight, this could be due to the high molecular weight of this protein, which increases the difficulty to achieve crystallization, but one has to note that crystallization has been already achieved for much bigger and less-studied proteins than fibronectin [for instance, it is the case of earthworm hemoglobin which has a $4 \times 10^{6} \mathrm{~g} / \mathrm{mol}$ molecular weight (39)]. In our opinion, a secondary consequence of our result is that the random conformation of fibronectin prevents crystallization.

\section{REFERENCES}

1. Ruoslahti, E. (1988) Annu. Rev. Biochem. 57, 375-413.

2. Potts, J. R., and Campbell, I. D. (1994) Curr. Opin. Cell. Biol. 6, 648-655

3. Tsyguelnaia, I., and Doolittle, R. F. (1998) J. Mol. Evol. 46, 612-614.

4. Yamada, K. M. (1989) in Fibronectin (Mosher, D. F., Ed.) pp 48-121, Academic Press, Inc., New York.

5. Kornblihtt, A. R., Pesce, C. G., Alonso, C. R., Cramer, P., Srebrow, A., Werbajh, S., and Muro, A. F. (1996) FASEB J. 10, 248-257.

6. Dickinson, C. D., Veerapandian, B., Dai, X.-P., Hamlin, R. C., Xuong, N.-H., Ruoslahti, E., and Ely, K. R. (1994) J. Mol. Biol. 236, 1079-1092.

7. Williams, M. J., Phan, I., Harvey, T. S., Rostagno, A., Gold, L. I., and Campbell, I. D. (1994) J. Mol. Biol. 235, 13021311 .

8. Sticht, H., Pickford, A. F., Potts, J. R., and Campbell, I. D. (1998) J. Mol. Biol. 276, 177-187.

9. Alexander, S. S., Colonna, G., Yamada, K. M., Pastan, I., and Edelhoch, H. (1978) J. Biol. Chem. 253, 5820-5824.

10. Alexander, S. S., Colonna, G., and Edelhoch, H. (1979) J. Biol. Chem. 254, 1501-1505.

11. Mosesson, M. W., Chen, A. B., and Huseby, R. M. (1975) Biochim. Biophys. Acta 386, 509-524.

12. Koteliansky, V. E., Glukhova, M. A., Bejanian, M. V., Smirnov, V. N., Filimonov, V. V., Zalite, O. M., and Venyaminov, S. Y. (1981) Eur. J. Biochem. 119, 619-624.

13. Odermatt, E., and Engel, J. (1989) in Fibronectin (Mosher, D. F., Ed.) pp 25-45, Academic Press, Inc., San Diego.

14. Edsall, J. T., Gilbert, G. A., and Scheraga, H. A. (1954) J. Am. Chem. Soc. 77, 157-161.

15. Williams, E. C., Janney, P. A., Ferry, J. D., and Mosher, D. F. (1982) J. Biol. Chem. 257, 14973-14978.

16. Rocco, M., Carson, M., Hantgan, R., McDonagh, J., and Heremans, J. (1983) J. Biol. Chem. 258, 14545-14549.

17. Rocco, M., Infusini, E., Daga, M. G., Gogioso, L., and Cuniberti, C. (1987) EMBO J. 6, 2343-2349.

18. Colonna, G., Alexander, S. S., Yamada, K. M., Pastan, I., and Edelhoch, H. (1978) J. Biol. Chem. 253, 7787-7790.

19. Sjöberg, B., Pap, S., Osterlund, E., Osterlund, K., Vuento, M., and Kjems, J. (1987) Arch. Biochem. Biophys. 255, 347-355; Sjöberg, B., Eriksson, M., Osterlund, E., Osterlund, K., and Pap, S. (1989) Eur. Biophys. J. 17, 5-11.

20. Engel, J., Odermatt, E., and Engel, A. (1981) J. Mol. Biol. 150, 97-120.

21. Price, T. M., Rudee, M. L., Pierschbacher, M., and Ruoslahti, E. (1982) Eur. J. Biochem. 129, 359-363.

22. Erickson H. P., Carell, N., and McDonagh, J. (1981) J. Cell. Biol. 91, 673-678.

23. Tooney, N. M., Mosesson, M. W., Amrani, D. L., Hainfeld, J. F., and Wall, J. S. (1983) J. Cell. Biol. 97, 1686-1692.

24. Zenhausern, F., Adrian, M., and Descouts, P. (1993) J. Electron Microsc. 42, 378-388.

25. Benecky, M. J., Wine, R. W., Kolvenbach, C. G., and Moseson, M. W. (1991) Biochemistry 30, 4298-4306.

26. Poulouin, L., Gallet, O., Rouahi, M., and Imhoff, J. M. (1999) Protein Expression Purif. 17, 146-152.

27. Mosesson, M. W., and Umfleet, R. A. (1970) J. Biol. Chem. $21,5728-5736$.

28. Higgins, J. S., and Benoît, H. C. (1994) Polymers and neutron scattering, Clarendon Press, Oxford.

29. Cotton, J.-P. (1991) in Neutron, X-ray and light scattering, (Linder, P., and Zemb, Th., Eds.) pp 3-31, North-Holland Publishing, Amsterdam.

30. Berne, B. J., and Pecora R. (1976) Dynamic light scattering with applications to chemistry, biology, and physics, Wiley, New York.

31. Yamakawa, H. (1971) Modern theory of polymer solutions, 
Harper \& Row Publishers, New York.

32. Oono, Y., and Kohmoto, M. (1983) J. Chem. Phys. 78, 520528.

33. Weill, G., and des Cloizeaux, J. (1979) J. Phys. 40, 99-105.

34. Jacrot, B. (1976) Rep. Prog. Phys. 39, 911-953.

35. Timmins, P. A., and Zaccai, G. (1988) Eur. Biophys. J. 15, 257-268.

36. Calmettes, P., Durand, D., Desmadril, M., Minard, P., Receveur, V., and Smith, J. C. (1994) Biophys. Chem. 53,
$105-114$.

37. Creighton, T. E. (1993) Proteins, 2nd ed., W. H. Freeman and Cie New York.

38. Leahy, D. J., Hendrickson, W. A., Aukhill, I., and Erickson, H. P. (1992) Science 258, 987-991.

39. Royer, W. E., Jr., and Hendrickson W. A. (1988) J. Biol. Chem. 27, 13762-13765.

BI992770X 